\title{
ASPECTOS MORFOLÓGICOS Y FISIOLÓGICOS DE HOBSONIA MIRABILIS \\ (PECK) LINDER, HIFOMICETO HELICOSPÓRICO REGISTRADO POR PRIMERA VEZ PARA MÉXICO
}

\author{
Gabriela Heredia Abarca \\ Rosa María Arias Mota \\ Y \\ Manuela Reyes Estebanez \\ Instituto de Ecología, A.C. \\ Apartado postal 63 \\ 91070 Xalapa, Veracruz \\ correo electrónico: heredia@ecologia.edu.mx
}

\section{RESUMEN}

Se registra por primera ocasión para México la especie Hobsonia mirabilis (Peck) Linder, hongo hifomiceto helicospórico saprobio, cuya característica primordial en el sustrato es la formación de pústulas blanquecinas con apariencia viscosa compuestas por esporodoquios. Se describe su morfología in situ y en los medios de cultivo más comúnmente usados en laboratorio; se anexan observaciones sobre su capacidad para crecer bajo diferentes fuentes de carbono.

Palabras clave: Hifomicetos, Hobsonia mirabilis, hongos helicospóricos, hongos conidiales.

\section{ABSTRACT}

Hobsonia mirabilis (Peck) Linder, a helicosporous fungus, is recorded for the first time from Mexico. This saprobic, leaf litter species forms white, gelatinous sporodochia on decaying vegetation. The fungus is described and illustrated in situ, and additional information on its morphology in culture is presented. Results of tests of its ability to grow on several carbon sources are also presented.

Key words: Hyphomycetes, Hobsonia mirabilis, conidial fungi, helicosporous fungi.

\section{INTRODUCCIÓN}

Los hifomicetos helicospóricos comprenden un grupo de hongos microscópicos relativamente pequeño; se caracterizan por producir conidios filiformes que adoptan formas semicirculares, circulares y helicoidales en uno o varios planos. Goos (1987) en su revisión sobre los hongos helicospóricos incluye 43 géneros, entre éstos, sólo unos cuantos producen sus conidios en esporodoquios, tal es el caso de Everhartia Saccardo 
Ellis, Vanbeverwijkia Agnihothrudu, Delortia Patouillard, Drepanoconis Schroeter Hennings apud Hennings, Troposporium Harkness, Troposporella Karsten y Hobsonia Berk.

A pesar de que el género Hobsonia fue descrito hace más de un siglo (Massee,1891), además de la especie tipo: Hobsonia mirabilis (Peck) Linder, únicamente se conocen dos taxa más: $H$. santessonii Lowen D. Hawksw. y H. christiansenii B. L. Brady D. Hawksw. Los hongos incluidos en este género forman colonias efusas, punctiformes, compuestas de esporodoquios de apariencia viscosa. Los conidióforos son micronematosos, poco diferenciados del micelio; los conidios se encuentran agregados en una sustancia mucilaginosa, están compuestos por un filamento septado hialino el cual se enrosca en varios planos, formando espirales que pueden adquirir formas caprichosas con apariencia de nudos esféricos. La ontogenia conidial es holoblástica, las esporas se originan en la parte apical de delgadas hifas espirales, el eje del conidio se encorva a medida que se extiende en longitud. Al madurar el conidio, se delimita de la célula conidiógena mediante la formación de un septo; la secesión ocurre en el punto de unión con la célula conidiógena.

Hobsonia mirabilis es una especie saprobia, se distribuye principalmente, pero no exclusivamente, en zonas tropicales. Existen citas de colectas procedentes de Venezuela, Panamá, Colombia, Hawai, Brasil y algunos sitios de Estados Unidos (Martin, 1959; Linder, 1929).

Para México son muy pocas las especies helicospóricas descritas, apenas suman 10 los registros publicados (Kohlmeyer, 1968; Mercado Sierra y Heredia, 1994; González y Herrera, 1995; Heredia et al., 1995). El único antecedente ex profeso sobre hongos helicospóricos es el de Arias et al. (2002), en el cual se describen para el estado de Veracruz las especies Helicoma ambiens, $H$. olivaceum, Helicosporium panacheum, $H$. talbotii y Helicomina trichophila.

En general, a nivel mundial, el conocimiento sobre los hongos helicospóricos se ha concentrado en aspectos taxonómicos, quedando al margen su biología y manipulación a nivel de laboratorio. Muy poco se conoce sobre su capacidad para desdoblar las principales moléculas estructurales vegetales, como son la celulosa y la hemicelulosa. El presente trabajo tiene como objetivos, además de registrar y describir por primera vez material de Hobsonia mirabilis colectado en México, aportar datos sobre su capacidad para desarrollarse in vitro en medios nutritivos con diferentes fuentes de carbono.

\section{METODOLOGÍA}

Para estimular la esporulación, el material colectado se colocó por un periodo entre 5 y 15 días en cámaras húmedas. A medida que surgieron las masas de esporodoquios, se extrajeron muestras para la elaboración de preparaciones microscópicas permanentes, para lo cual se utilizó como medio de montaje alcohol polivinílico; paralelamente se tomaron secciones de los esporodoquios, las cuales fueron sombreadas con oro paladio por 15 minutos con el fin de observarlas a través del microscopio electrónico de barrido (microscopio JEOL Mad JSM-T20).

Mediante el uso de un micromanipulador se aisló el hongo y se obtuvieron cepas monospóricas. Para conocer la morfología de sus colonias in vitro se inocularon los aislamientos en los medios papa dextrosa agar (PDA), extracto de malta agar (EMA) y 
maíz agar; periódicamente se registraron las características de las colonias. Asimismo se comparó la capacidad del hongo para crecer en medios con diferentes fuentes de carbón como son almidón, celulosa, alfa-celulosa, carboximetilcelulosa y xilanos, todos estos son polímeros estructurales de las paredes vegetales. En esta ocasión se inoculó el hongo en el centro de las placas de agar con las diferentes fuentes de carbón y se midió diariamente el diámetro de la colonia hasta que se cubrieron las cajas. En todos los casos las placas fueron incubadas a $25^{\circ} \mathrm{C}$ en la oscuridad. El material de referencia se encuentra depositado en la colección de hongos del Instituto de Ecología A.C. (XAL) y en el cepario del departamento de Biología de Suelos del mismo instituto.

\section{DESCRIPCIÓN TAXONÓMICA IN SITU}

Hobsonia mirabilis (Peck) Linder, Brittonia 11: 98-101. 1959. Figs. 1-9.

= Hobsonia gigaspora Berk.

= Hobsonia ackermanni Patouillard

Colonias errumpentes, punctiformes, a veces confluentes, formadas por esporodoquios de 0.5 a $3.0 \mathrm{~mm}$ de ancho, circulares, ovoides o de forma irregular, blanquecinas cuando inmaduras, posteriormente con tonalidades amarillentas, de apariencia viscosa, gelatinosa. Conidióforos micronematosos, hialinos, lisos, delgados, de 1.8-3 $\mu \mathrm{m}$ de grosor, longitud variable, en la base rectos y helicoidales en la parte media y apical. Células conidiógenas integradas, monoblásticas, variables en talla, secesión esquizolítica. Conidios dispuestos en agregados embebidos en un mucílago, hialinos, filamentosos, transversalmente septados, helicoidales, tridimensionales, formados por $1^{\circ}$ la $3^{\circ}$ hélices, con paredes lisas, los filamentos tienen un grosor de 9-14 $\mu \mathrm{m}$. Los conidios maduros alcanzan hasta $46 \mu \mathrm{m}$ de diámetro en su parte más ancha.

Material examinado y sustrato: VERACRUZ. Cuautolontitla, municipio Calcahualco. CB714, 10/08/01. Bosque mesófilo, sobre hojarasca. VERACRUZ. Cascadas de Texolo, municipio Xicochimalco. CB715, 23/06/01. Vegetación secundaria, sobre hojas de encino.

Observaciones. El material estudiado coincide tanto en la morfología como en las dimensiones de los conidios, con las características de Hobsonia mirabilis. Las otras dos especies descritas en el género fácilmente pueden diferenciarse de $H$. mirabilis; $H$. christiansenii forma esporodoquios de color rosa intenso y los conidios tienen conspicuas constricciones en los septos, en tanto que en $H$. santessonii los esporodoquios presentan tonalidades rosadas, grisáceas o amarillentas y los conidios se caracterizan por la presencia de verrugas oscuras en su superficie (Lowen et al., 1986).

Descripción de las colonias in vitro. En PDA y EMA el crecimiento es similar; a partir del segundo día se observan hifas, entre el tercer y cuarto día del centro de la colonia y en forma radial se forman delgados cordones blanquecinos, de los cuales surgen numerosas ramificaciones laterales compuestas de hifas hialinas mayormente sumergidas en el sustrato; a medida que la colonia crece la disposición peculiar de los cordones le confiere una apariencia semejante a un rehilete. En ambos casos el micelio 

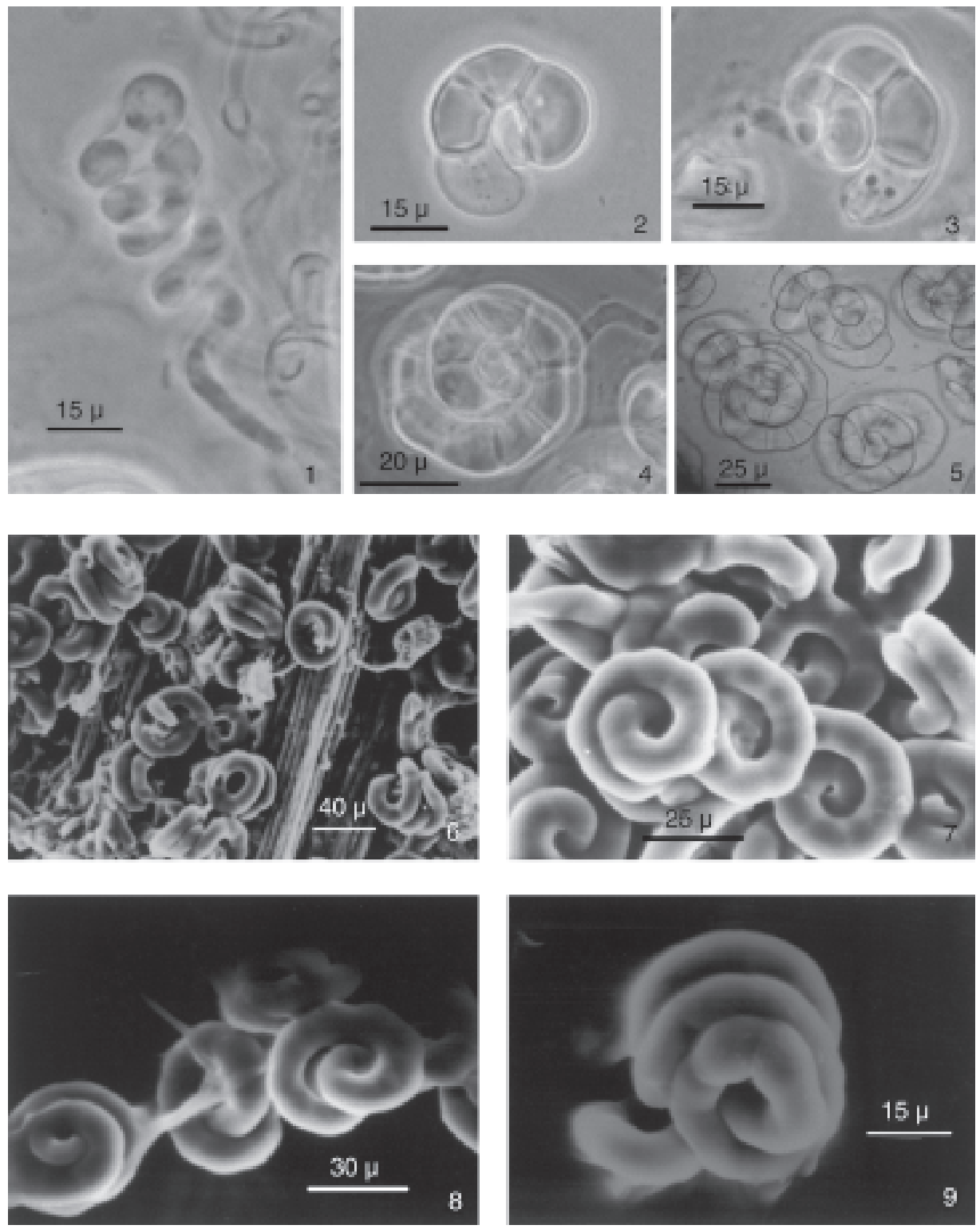

Hobsonia mirabilis. 1. Conidióforo helicoidal con primordio de conidio. 2-4. Diferentes estados de desarrollo conidial. 2. Conidio joven en un plano. 3. Conidio con dos hélices adherido al conidióforo. 4. Conidio casi maduro aun adherido al conidióforo. 5. Conidios maduros con tres hélices. 6-7. Esporodoquio sobre hojas de Quercus sp. 8-9. Conidios con restos de mucílago. 
Heredia et al.: Aspectos morfológicos y fisiológicos de Hobsonia mirabilis
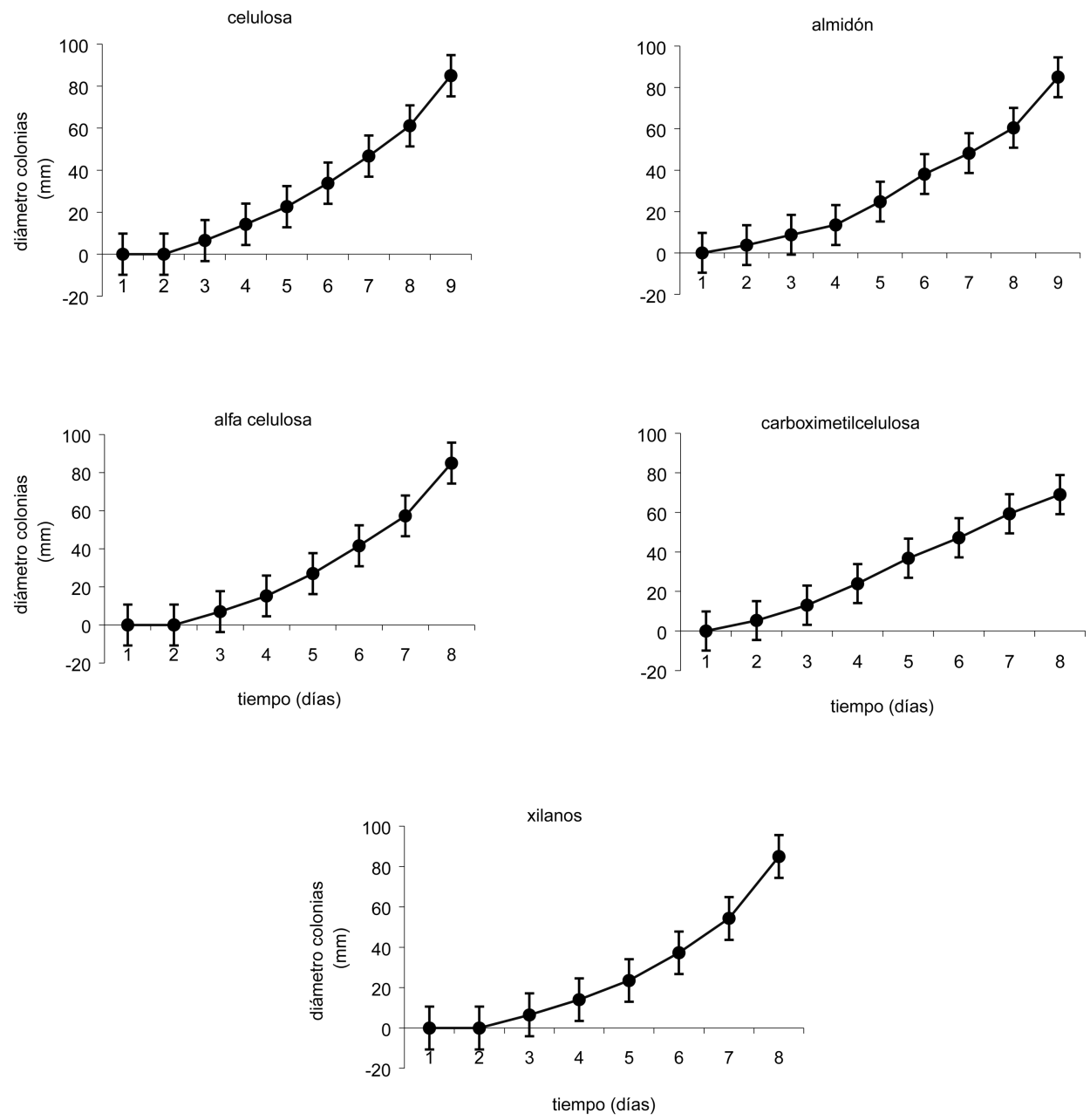

Fig. 10. Crecimiento de Hobsonia mirabilis en placas de agar con diferentes fuentes de carbono. Cada dato corresponde al promedio de cinco repeticiones. 
es escaso; a los ocho días las colonias cubren totalmente la superficie de la caja, son blanquecinas y hialinas al reverso, sin que se aprecie ningún cambio en la coloración del medio.

En PDA a los 10 días aparecieron primordios de conidióforos, a los 17 días se detectó la formación de conidios sin que se formaran esporodoquios. En EMA, por el contrario, a los 10 días se desarrollaron en la parte media de las colonias esporodoquios blanquecinos con apariencia gelatinosa, semejantes a los observados sobre el sustrato in situ. En el medio maíz agar, el hongo se extendió más rápidamente, a los siete días las cajas estaban completamente cubiertas; no obstante, en este caso la formación del micelio fue escasa y se caracterizó por el desarrollo de largas hifas delgadas, hialinas, con pocas ramificaciones. En este medio no se detectó esporulación.

Desarrollo en diferentes fuentes de carbono. En la Fig. 10 se puede observar que $H$. mirabilis creció en las cinco fuentes de carbono probadas. Entre el octavo y el noveno día todas las colonias cubrieron las placas; en todos los casos la morfología y disposición de las colonias fue similar. Las colonias se caracterizaron por presentar tonalidades blanquecinas con micelio poco denso e inmerso en el sustrato.

La capacidad de los hongos saprobios de la hojarasca para hidrolizar moléculas complejas es un factor importante para la obtención de energía. La celulosa, la hemicelulosa y sus componentes son los polisacáridos estructurales más abundantes en los tejidos foliares, su hidrólisis requiere una serie de reacciones bioquímicas las cuales son catalizadas por complejos enzimáticos (Dix y Webster, 1995). Con excepción de algunos hongos basidiomicetos, causantes de pudriciones blandas en madera, son muy pocas las especies que producen todas las enzimas necesarias para llevar a cabo en su totalidad la celulolisis. La habilidad de algunos micromicetos para hidrolizar xilanos, celulosa y carboximeticelulosa ha sido probada en hongos filamentosos aislados del suelo (Domsch y Gams, 1960) y de restos vegetales agrícolas (Flannigan, 1970). En estos trabajos se ha constatado que existe una amplia gama en la respuesta de los hongos filamentosos para depolimerizar las cadenas de polisacáridos. Al respecto no existe información sobre las especies fúngicas de la hojarasca y los datos obtenidos en el ensayo realizado sugieren que Hobsonia mirabilis tiene la capacidad para aprovechar las fuentes de carbono probadas, lo que le confiere la posibilidad de sintetizar al menos algún tipo de enzimas involucradas en la descomposición de la celulosa y hemicelulosa. Estos datos dan la pauta para el planteamiento de futuros estudios de actividad enzimática, que en forma cualitativa revelen el potencial degradador de la especie estudiada.

\section{AGRADECIMIENTOS}

Se agradece la colaboración del Sr. Tiburcio Láez en la preparación y observación del material al microscopio de barrido. Esta contribución fue realizada con el apoyo del proyecto 902-07 del Instituto de Ecología A.C. 


\section{LITERATURA CITADA}

Arias, R. M., G. Heredia y M. Reyes. 2002. Primer registro para México de cinco especies de hongos conidiales helicospóricos. Rev. Mex. Mic. 16: 12-18.

Dix, N. J. y J. Webster. 1995. Fungal ecology. Chapman \& Hall. Londres. 497 pp.

Domsch, K. H. y W. Gams. 1960. Variability and potential of a soil fungus population to decompose pectin, xylan and carboxymethyl cellulose. Soil Biol. Biochem. 1: 29-36.

Flannigan, B. 1970. Degradation of arabinoxylan and carboxymethyl cellulose by fungi isolated from barley kernels. Trans. Br. Mycol. Soc. 55: 277-281.

González, M. C. y T. Herrera. 1995. Micromicetos marinos lignícolas de la laguna costera Barra de Navidad, estado de Jalisco, México. Rev. Mex. Mic. 11: 145-154.

Goos, R. D. 1987. Fungi with a twist: the helicosporous hyphomycetes. Mycologia 79: 1-22.

Heredia, G., A. Mercado-Sierra y J. Mena-Portales. 1995. Conidial fungi from leaf litter in a mesophilic cloud forest of Veracruz, Mexico. Mycotaxon 55: 473-490.

Kohlmeyer, J. 1968. Marine fungi from the tropics. Mycologia 60: 252-269.

Linder, D. H. 1929. A monograph of the helicosporous fungi. Ann. Missouri Bot. Gard. 16: 227-388.

Lowen, R., Brady B. L., D. L. Hawksworth y R. R. M. Paterson. 1986. Two new lichenicolous species of Hobsonia. Mycologia 78(5): 842-846.

Martin, G. W. 1959. On the genus Hobsonia. Brittonia 11: 98-101.

Massee, G. 1891. A new genus of Tubercularieae - Hobsonia. Ann. Bot. (London) 5: 509.

Mercado-Sierra, A. y G. Heredia. 1994. Hyphomycetes asociados a restos vegetales en el estado de Veracruz. Rev. Mex. Mic. 10: 33-48. 